\title{
A Fast Stitching Algorithm for Generating Panorama on Mobile Intelligent Terminal
}

\author{
Guangyu Bian \\ School of Computer Science and Technology, Beijing \\ University of Posts and Telecommunications \\ Beijing 100876, China \\ bgy2011211237@bupt.edu.cn
}

\begin{abstract}
This article proposes an improved stitching algorithm based on grayscale to generate panorama. We adopted Moravec operator to extract the feature point and simplified the extraction and image fusion process to downsize the scale of calculation. Meanwhile, with the utilization of noise reduction, exposure processing and mismatch correction, the quality of the panorama and the robustness of the algorithm was well ensured. Tests show that this algorithm is practical for low-speed mobile terminals and it is considerably efficient and not sensitive about illumination condition or slight displacement during shooting.
\end{abstract}

Keywords-Image mosaicing, Mobile Intelligent Terminal, Moravec operator, Featured area matching

\section{INTRODUCTION}

In recent years, the technology of image rendering was proposed and developed to generate virtual scene with photorealistic. Panorama is one of the most significant major research direction which is widely utilized in virtual environment, computer vision and multimedia. Nowadays there are two methods to generate panorama: direct mode and image stitching. The former mode is quite costly due to the requirement of panoramic camera. The latter method is much more feasible and acceptable by preprocessing, mapping and merging the original image took by ordinary camera[1].

Under normal circumstances, it is difficult to balance the tradeoffs between the speed of processing and the precision of the algorithm. That is, the high-accuracy algorithm is not suitable for low-speed device situation while algorithm with a small scale of calculation is too sensitive about the quality of the original image. This article proposed a new fast- stitching algorithm which is not resource consuming or too sensitive about light condition, slight displacement and rotation during shooting. It shows reasonable robustness.

\section{IMAGE PREPROCESSING}

In the article, the preprocessing is of great significance to downsizing the calculation and ensuring the accuracy and quality. There are 3 steps in this method: image noise reduction, cylindrical projection and exposure processing.

\author{
Dengkui Zhang \\ School of Computer Science and Technology, Beijing \\ University of Posts and Telecommunications \\ Beijing 100876, China \\ balloonzhang@163.com
}

\section{A. Image Noise Reduction}

The noise of image is known as the possible information that may leading to misunderstanding or confusion. It happens unpredictably and randomly. Pixel with large deviation is called the noise point which has great influences on the analysis and process of the image. This article employs gray-level-difference-based Moravec operator to extract the feature point of the image. Hence, it's necessary to reduce the image noise. Median filtering method[2] is applied here as follows:.

$$
g(x, y)=\operatorname{Med}\{f(x \pm a, y \pm b), a \in W, b \in W\}
$$

$f(x, y)$ and $g(x, y)$ is the image before and after processing. $\mathrm{W}$ is a two-dimensional template of $3 \times 3$ or $5 \times 5$ of a figure of line, circle, cross, ring, etc. We use a square of $3 \times 3$ as the template to downsize the calculation and speed the processing up.

\section{B. Cylindrical Projection}

The section of images took during the rotation of the camera about the optical center appear various shape due to view angle, that is, the image distortion. Adopting these images can led to large deviation and unacceptable visual effect. In this way, cylindrical projection is indispensable.

The relationship between Cartesian coordinate system and Cylindrical coordinate system is the way we transform each pixel. See Fig.1.

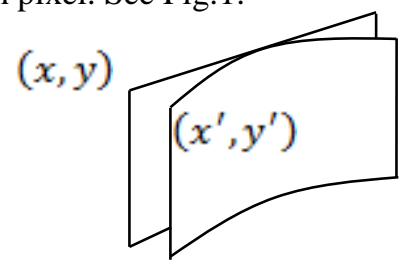

Figure 1. Tow coordinate systems.

Step 1: Calculating the focal distance $f$ of the camera by the average offset between adjacent photos in the shooting section.

$$
f=\sum_{k=1}^{n} \frac{a_{k}}{2 \pi}
$$


$a_{k}$ is the offset between image No. $k$ and No. $k+1$ determined by phase correlation method[3]. $n$ is the amount of the photos.

Step 2: Calculating the coordinate of each pixel utilizing the following transforming formula [4]. See Fig.2.

$$
\begin{aligned}
& x^{r}=f \times \tan ^{-1} \frac{x-\frac{w}{2}}{f}+f \times \tan ^{-1} \frac{w}{2 f} \\
& \dot{y}^{I}=\frac{f \times\left(y-\frac{H}{2}\right)}{\sqrt{\left(x-\frac{w}{2}\right)^{2}+f^{2}}}+\frac{H}{2}
\end{aligned}
$$
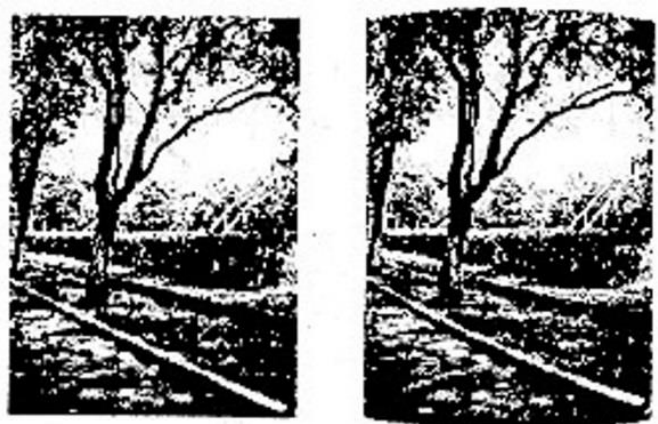

Figure 2. Image before and after projection.

\section{Exposure Processing}

With different view angle, the illumination intensity differs. The changing of the exposure of the adjacent film gives the viewer a feeling of incompleteness and lack of realness because of the brightness mismatch. Homomorphic filtering[5]is the most common way to solve this problem by adjusting the gray level difference according to the light intensity and reflectivity of the image. However, this algorithm is of unreasonable complexity for common equipment. Therefore, we employed a simpler method to balance the brightness of adjacent images.

According to the average offset between adjacent photos, we can determine the area of the overlapping portion of two images, setting as $M_{1}$ and $M_{2}$, the average gray level difference of them is $S_{1}$ and $S_{2}$. We use the gray scale ratio $\alpha=\frac{s_{1}}{s_{2}}$ to balance the brightness of these images.

\section{IMAGE REGISTRATION}

After preprocessing the images, we narrow the problem down as matching the corresponding coordinates in overlapping area. There are three major methods: frequency-domain transform, template-based enumeration search, feature-based matching. The first and the fastest one is not stable enough to ensure a reasonable output. The second and the most accurate one is too resource- consuming. In this case, we applied the last method as a compromise of efficiency and quality. There are 3 main steps: extracting feature points, matching feature points and correcting the mismatch.

\section{A. Extracting Feature Point}

Feature points are pixels having the characteristic information of the image, that is, the manifest point such as the corner of the table and the end of the brunch, etc.

In this article we applied gray-level-difference-based Moravec operator[6]to extract the feature point of the image. By evaluating the grayscale difference variance, the interest values, between a certain area and its neighboring area, we can determine the feature point as the local maxima of the interest value with non-maximum suppression.

For instance, to calculate the interest value of $A_{5}$, we select a $3 \times 3$ template. Then we compute the gray level difference after moving the template horizontally vertically and diagonally for a unit. In Fig.3, template was moved to the right-top direction. Its gray level difference is:

$$
\sigma=\sum_{i=1}^{9}\left(B_{i}-A_{i}\right)^{2}
$$

We chose the minimum of $\sigma$ of all 8 directions as the interest value of $A_{5}$. Afterwards, we determined the local maxima of the interest value with non-maximum suppression as the feature point. As in the image, feature point is a corner pixel with gray level difference of a large scale.

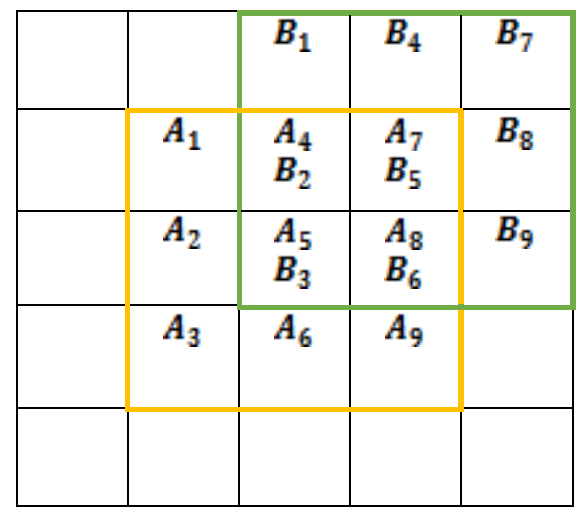

Figure 3. Moravec operator.

\section{B. Matching Feature Points}

After feature points extraction, we need to match the corresponding points of the adjacent images. Mostly, template-enumerating is used to match which gives accurate output and requires a large cost of calculation. This article utilized the cross correlation coefficient of grayscale of the feature points to match them. 
For each feature point, we select the surrounding $7 \times 7$ area as the correlation window. We normalize the points to eliminate the effects of brightness and compute the cross correlation coefficient of each pair of feature points with the following formula:

$$
R=\frac{\sum_{x, y \in W}\left[G_{1}(x, y)-\overline{G_{1}}\right]\left[G_{2}(x, y)-\overline{G_{2}}\right]}{\sqrt{\sum_{x y \in W}\left[G_{1}(x, y)-\overline{G_{1}}\right]^{2}} \sqrt{\sum_{x, y \in W}\left[G_{2}(x, y)-\overline{G_{2}}\right]^{2}}}
$$

$\mathrm{W}$ is the correlation window. $G_{1}, G_{2}$ and $\overline{G_{1}}, \overline{G_{2}}$ is the actual and average value of the grayscale of the feature point in two images. Finally, we choose the points with maximum cross correlation coefficient $R$ as corresponding points.

\section{Matching Feature Points}

By adopting the algorithm below, the ratio of mismatch is acceptable. However, we can't afford the damage that certain mismatch of important feature points may occur. Hence, the correction of mismatch is needed to guarantee the robustness the algorithm and the quality of final panorama.

To ensure the efficiency of the algorithm, we applied the vector detection, more efficient and practical than other complicated detection method.

Assuming that there are two pairs of feature points: $P_{1}$ $P_{2}$ and $Q_{1}-Q_{2}$. Thus the discrepancy of their feature vectors $\overrightarrow{N_{1}}=P_{1}-P_{2}$ and $\overrightarrow{N_{2}}=Q_{1}-Q_{2}$ is negligible. Otherwise, these feature points were mismatched. Re-calculation and rematch is necessarily needed.

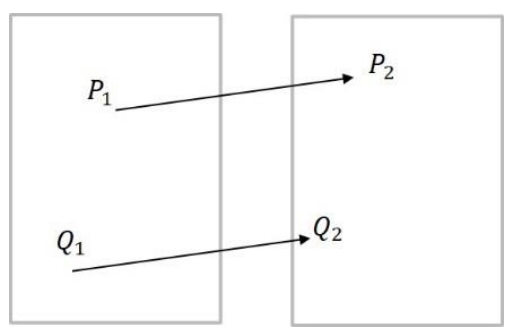

Figure 4. Feature vector.

\section{IMAGE FUSION}

After matching the feature points and vectors, we can determine the transforming relationship of corresponding coordinates. To Stitch the images perfectly, the slight displacement and rotation during shooting should be eliminated as well as the overlapping area of the adjacent image.

Mean fusion is the most common method of image fusion by applying the average grayscale of the overlapping area which leads to the problem of double image and blurring. In this article, we applied the weighted function [7] to deal with the geometric distortion during the image fusing process.
Setting $G_{1}(x, y)$ and $G_{2}(x, y)$ as the grayscale of the overlapping area of two images, the grayscale after fusion is determine as follows:

$$
G_{0}(x, y)=\alpha G_{1}(x, y)+(1-\alpha) G_{2}(x, y)
$$

$\alpha$ is the weighting factor. According to our experiment, the algorithm gives the best output when $\alpha$ is $x /$ width in which $x$ is the abscissa of the overlapping area, width is the maximum value of the abscissa regarding the leftbottom as the origin.

Moreover, if the difference between $G_{1}(x, y)$ and $G_{2}(x, y)$ is larger than a certain threshold, the output of this algorithm has the problem of double image. In this case, we apply the value of $G_{1}(x, y)$ or $G_{2}(x, y)$ as $G_{0}(x, y)$.

\section{CONCLUSION}

We tested our algorithm on several low-speed mobile devices by taking a section of photos as Fig.5-8.

We utilized an Android mobile phone to run the algorithm to get the following result as Fig.9.

Moreover, we tested the algorithm under different illumination and view conditions for several times and we got a large amount of considerable final panorama which proved the robustness and feasibility of this algorithm.

This article improved the common stitching algorithm based on grayscale to generate panorama. With appropriate simplification and improvement, we downsized the feature point extraction and image fusion process as well as the scale of calculation. Meanwhile, Utilizing noise reduction, exposure processing and mismatch correction, the quality of the panorama and the robustness of the algorithm was well improved.

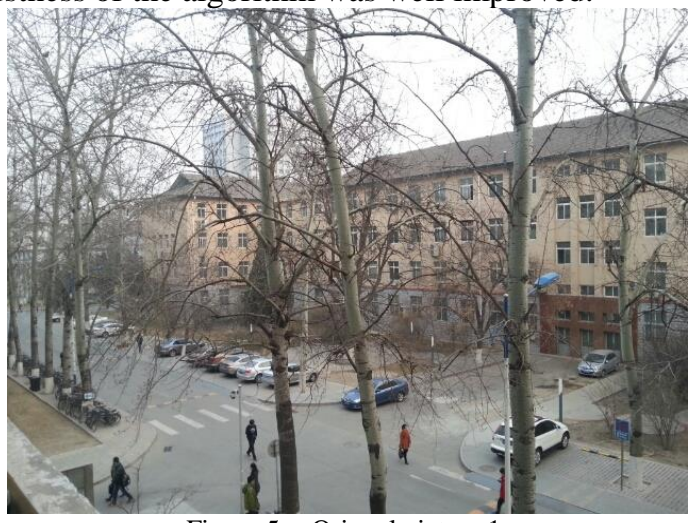

Figure 5. Orignal picture 1. 


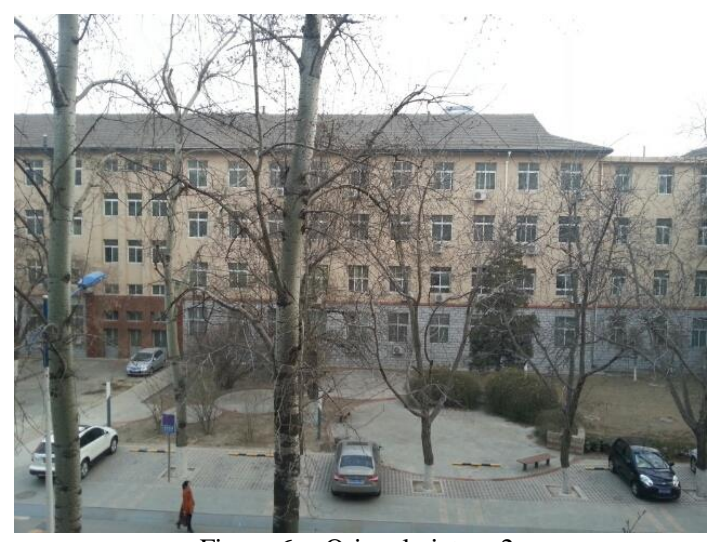

Figure 6. Orignal picture 2.



Figure 7. Orignal picture 2.

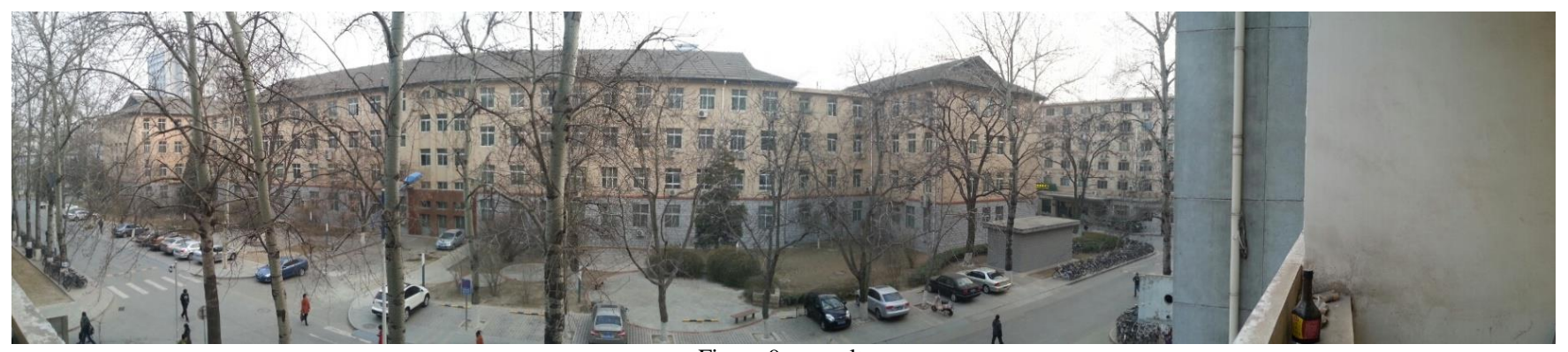

Figure 9. result.

\section{REFERENCES}

[1] He Panli, Fan Tiesheng, "Image Mosaic of Improved Algorithm Based on Gray Scale," Journal of Shenyang University, April 2006, vol.18, No.2, pp.37-39.

[2] Sun Hongqi, Shi Weiying, Ju Yongfeng, "Image Processing with Medium Value Filter" Journal of Chang'an University, Mar 2003, vol.23, No.2, pp.104-106

[3] M.H.G.Peeters, "Implementation of the Phase Correlation Algorithm," Faculty of Electrical Engineering Section Design Technology For Electronic Systems (ICS/ES)

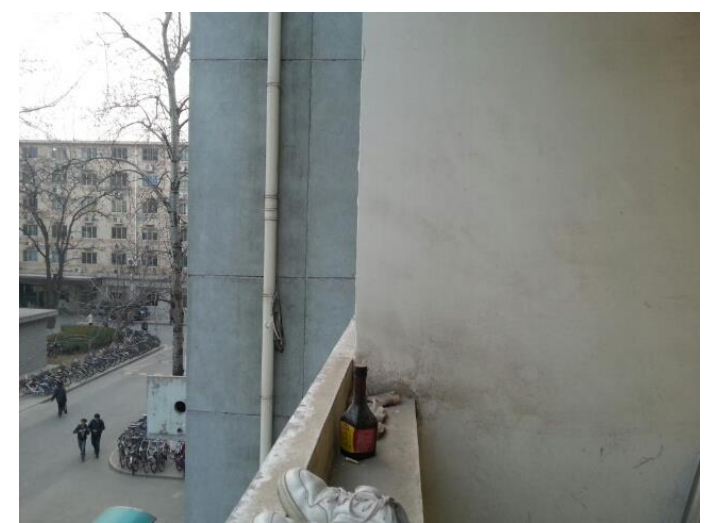

Figure 8. Orignal picture 2.

[4] Feng Jingbo, Su Zhixun, Liu Xiuping, "An Similar-Curve-Based Automatic Panoramic Image Mosaic," Journal of Computers, Nov 2003, pp.1604-1608,

[5] Delac, K., Grgic, M., \& Kos, T. "Sub-image homomorphic filtering technique for improving facial identification under difficult illumination conditions". In International Conference on Systems, Signals and Image Processing, September 2006, Vol. 1, pp. 21-23.

[6] Förstner, W., \& Gülch, E. "A fast operator for detection and precise 1 ocation of distinct points, corners and centres of circular features. In P roc. ISPRS intercommission conference on fast processing of photogr ammetric data, June 1987, pp. 281-305.

[7] Hu Shejiao, Ge Xiwang, Su Ling, "Panoramic image mosaic based on domain correlation," Journal of Hefei University of Technology, Jan.2007, Vol.30, No.1, pp.29-32. 